\title{
Contribution of hyperglycemia on diabetic complications in obese type 2 diabetic SDT fatty rats: effects of SGLT inhibitor phlorizin
}

\author{
Yoshiaki KATSUDA ${ }^{1)}$, Tomohiko SASASE ${ }^{1)}$, Hironobu TADAKI ${ }^{1)}$, Yasuko MERA ${ }^{1)}$, \\ Yu MOTOHASHI ${ }^{1)}$, Yusuke KEMMOCHI'), Kaoru TOYODA ${ }^{2)}$, Kochi KAKIMOTO ${ }^{2)}$, \\ Shinichi $\mathrm{KUME}^{3)}$, and Takeshi OHTA ${ }^{1)}$ \\ 1) Biological/Pharmacological Research Laboratories, Central Pharmaceutical Research Institute, Japan Tobacco \\ Inc., 1-1 Murasaki-cho, Takatsuki, Osaka 569-1125, Japan \\ 2) Toxicology Research Laboratories, Central Pharmaceutical Research Institute, Japan Tobacco Inc., 23 Naganuki, \\ Hadano, Kanagawa 257-0024, Japan \\ ${ }^{3)}$ Graduate School of Agriculture, Kyoto University, Kitashirakawa Oiwake-cho, Sakyo-ku, Kyoto 606-8502, Japan
}

\begin{abstract}
The spontaneously diabetic torii (SDT) fatty rat is a new model of type 2 diabetes showing overt obesity, hyperglycemia and hyperlipidemia. With early onset of diabetes mellitus, diabetic microvascular complications, including nephropathy, peripheral neuropathy and retinopathy, are observed at young ages. In the present study, blood glucose levels of female SDT fatty rats were controlled with phlorizin, a non-selective SGLT inhibitor, to examine whether and how these complications are caused by hyperglycemia. Phlorizin treatment adequately controlled plasma glucose levels during the experiment. At 29 weeks of age, urinary albumin excretion considerably increased in SDT fatty rats. Glomerulosclerosis and tubular pathological findings also indicate diabetic nephropathy. These renal parameters tended to decrease with phlorizin; however, effects were partial. Sciatic nerve conduction velocities were significantly delayed in SDT fatty rats compared with SpragueDawley (SD) rats. Intraepidermal nerve fiber density, an indicator of subclinical small nerve fiber neuropathy, significantly decreased in SDT fatty rats. Retinal dysfunction (prolongation of peak latency for oscillatory potential in electroretinograms) and histopathological eye abnormalities, including retinal folding and mature cataracts were also observed. Both nerve and eye disorders were prevented with phlorizin. These findings indicate that severe hyperglycemia mainly causes diabetic complications in SDT fatty rats. However, other factors, such as hyperlipidemia and hypertension, may affect diabetic nephropathy. These characteristics of diabetic complications will become helpful in evaluating new drugs for diabetic complications using SDT fatty rats.
\end{abstract}

Key words: diabetic complications, nephropathy, peripheral neuropathy, retinopathy, SDT fatty rat

\section{Introduction}

Diabetes mellitus is one of the most common metabolic disorders, and the number of diabetic patients has been increasing worldwide. The International Diabetes Federation (IDF) reported that the 366 million patients with diabetes in 2011 will increase to 552 million by 2030 [11]. Even worse, more than half of all diabetics have one or more diabetic microvascular complications, such as diabetic nephropathy (DN), diabetic peripheral neuropathy (DPN) or diabetic retinopathy (DR), all of which seriously threaten quality of life. To clarify the

(Received 29 September 2014 / Accepted 11 November 2014 / Published online in J-STAGE 22 January 2015)

Address corresponding: T. Sasase, Biological/Pharmacological Research Laboratories, Central Pharmaceutical Research Institute, Japan Tobacco Inc., 1-1 Murasaki-cho, Takatsuki, Osaka 569-1125, Japan

(C)2015 Japanese Association for Laboratory Animal Science 
pathogenetic mechanism of human diabetes and its complications, and to develop drugs for diabetes, experimental diabetic animal models play critical roles.

The spontaneously diabetic torii (SDT) fatty rat is a new model for obese type 2 diabetes established by introducing the $f a$ allele of the Zucker fatty rat into the original (non-obese) SDT rat genome to defect leptin receptor signaling. Since SDT fatty rats develop marked hyperglycemia with hyperinsulinemia, hyperlipidemia and hypertension shortly after weaning [12-14, 17, 19, 23], SDT fatty rats develop severe microvascular complications at an early age $[17,19,20]$. Therefore, this animal model is useful for investigating diabetic complications and for evaluating new drugs. Previously, we investigated diabetic microvascular complications in original SDT rats by controlling blood glucose level with insulin treatment and showed that complications are caused by severe hyperglycemia $[25,26]$. However, because of hyperinsulinemia associated with marked insulin resistance, insulin treatment failed to control blood glucose level in SDT fatty rats (unpublished data). Therefore in the present study, we investigated diabetic complications by controlling blood glucose level with daily phlorizin (PZN) treatment. Phlorizin is a natural compound originally isolated from apple trees [3]. Its pharmacological mechanism is inhibiting sodium glucose co-transporters (SGLTs) distributed in the proximal tubule brush border (SGLT2) and gastrointestinal tract (SGLT1), leading to renal glucosuria and blocking intestinal glucose absorption, both of which reduce hyperglycemia [1]. In anticipation of these mechanisms, we administered phlorizin to SDT fatty rats to control blood glucose level and studied whether and how hyperglycemia causes diabetic microvascular complications in this model.

\section{Materials and Methods}

\section{Animals and chemicals}

Female SDT fatty rats from our colony were used in the study. At six weeks of age, SDT fatty rats were divided into two groups $(\mathrm{n}=8)$; a phlorizin treated group and a vehicle treated group. Age-matched female Sprague-Dawley (SD) rats (Charles River Laboratories Japan, Yokohama, Japan) were used as control animals $(\mathrm{n}=8)$. All animal protocols used in the study were in strict compliance with our own Laboratory Guidelines for Animal Experimentation. Animals were housed in a climate-controlled room (temperature $23 \pm 3^{\circ} \mathrm{C}$, humid- ity $55 \pm 15 \%, 12 \mathrm{~h}$ lighting cycle) and allowed free access to basal diet (CRF-1, Oriental Yeast, Tokyo, Japan) and water.

Phlorizin (Kanto chemical, Tokyo, Japan) was suspended in $20 \%$ propylene glycol and injected subcutaneously once daily $(100 \mathrm{mg} / \mathrm{kg} /$ day $)$ to animals in the phlorizin treated group for 23 weeks. Twenty \% propylene glycol was administered to animals in the vehicle treated group and control SD rats.

\section{Biochemical parameters}

During the experimental period, biochemical parameters were monitored. Blood samples were collected from the tail vein under fed condition. Glucose, $\mathrm{HbAlc}$, triglycerides (TG), free fatty acid (FFA) and total cholesterol (TC) were measured using commercial kits (Roche Diagnostics, Basel, Switzerland) and an automatic analyzer (Hitachi 7180; Hitachi High-Technologies, Tokyo, Japan). Commercial ELISA kits were used to measure plasma insulin (Rat Insulin ELISA Kit; Morinaga Institute of Biological Science, Yokohama, Japan).

\section{Evaluation of diabetic nephropathy}

Urine samples were collected for $24 \mathrm{~h}$ using metabolic cages. During urine sampling, animals were not limited to access to diet and water. Urinary glucose level was measured as described above. Both urinary and plasma creatinine levels were measured with an automatic analyzer to calculate creatinine clearance. Urinary albumin (Nephrat II; Exocell, Philadelphia, PA, USA) and urinary 8-OHdG (New 8-OHdG Check ELISA; Japan Institute for the Control of Aging, Nikken SEIL, Shizuoka, Japan) were measured using commercial kits.

\section{Evaluation of peripheral neuropathy}

Nerve conduction velocity was measured in accordance with previously described methods [29]. Briefly, the sciatic nerve was stimulated at the sciatic notch and the Achilles tendon using adequate intensity under 37.5 $\mathrm{mg} / \mathrm{kg}$ of sodium pentobarbital (Kanto chemical) and 3 $\mathrm{mg} / \mathrm{kg}$ of diazepam anesthesia. Action potentials in the muscle were recorded via PowerLab through a needle electrode. Motor nerve conduction velocity (MNCV) was calculated from the delta latency between M-wave peaks divided by the distance of the nerve length measured. Sensory nerve conduction velocity (SNCV) was also calculated from F-wave peaks. 


\section{Evaluation of retinopathy and cataracts}

Electroretinograms (ERGs) were performed as previously described [26] with slight modifications. Briefly, rats were adapted to darkness for at least $60 \mathrm{~min}$ and anesthetized with an intraperitoneal injection of $37.5 \mathrm{mg} /$ kg ketamine (Daiichi Sankyo Propharma, Tokyo, Japan) and $4.5 \mathrm{mg} / \mathrm{kg}$ xylazine hydrochloride (MP Biomedicals, Santa Ana, CA, USA). A 40 J xenon lamp was flashed after pupillary mydriasis with $0.5 \%$ tropicamide (Mydrin P; Santen, Osaka, Japan) and potential was recorded via the PowerLab data acquisition system and software Scope (ADInstruments, Dunedin, New Zealand) through a corneal contact lens electrode (Mayo, Aichi, Japan). Peak latencies of oscillatory potentials $\left(\mathrm{OP}_{1}, \mathrm{OP}_{2}\right.$ and $\mathrm{OP}_{3}$ ) were measured and data was expressed as the sum of $\mathrm{OP}_{1}$ to $\mathrm{OP}_{3}\left(\Sigma\left(\mathrm{OP}_{1}-\mathrm{OP}_{3}\right)\right)$.

Cataracts were evaluated after ERG measurements using a slit lamp (SL-14, Kowa, Tokyo, Japan). Lens opacity was scored using three grades for each eye as follows: 0 (no opacity; clear lens), 1 (partially clouded lens) and 2 (mature cataract; completely opaque lens). The average score of both eyes was used as the individual cataract score.

\section{Histology and immunohistochemistry}

At the end of the study, all animals were sacrificed at non-fasted condition by exsanguination under isoflurane anesthesia and necropsy was performed. Kidneys and eyes were fixed in 4\% paraformaldehyde (PFA) and 4\% glutaraldehyde/10\% neutral-buffered formalin, respectively. After resection, tissues were paraffin-embedded using standard techniques and thin-sectioned (3 to $5 \mu \mathrm{m}$ ). The sections were stained with hematoxylin and eosin (HE) for histological evaluation. Intraepidermal nerve fiber density (IENFD) was measured to evaluate small fiber neuropathy [15]. The skin of hind limbs was dissected and fixed in 4\% PFA, embedded in paraffin, and sectioned $(25 \mu \mathrm{m})$. Nerve fibers were immunostained for protein gene product 9.5 (PGP 9.5; rabbit polyclonal, 1:500; UltraClone, Isle of Wight, U.K.) overnight at $4^{\circ} \mathrm{C}$ and Alexa Fluor ${ }^{\circledR}$ mouse anti-rabbit IgG antibody 488 (1:1,000, Thermo Fisher Scientific, Waltham, MA, USA) for $30 \mathrm{~min}$ at room temperature. Five fields from each section were randomly selected and Z-stack images were obtained using a Nikon A1 confocal laser scanning microscope mounted on an inverted microscope (Eclipse Ti, Nikon, Tokyo, Japan). Nerve fibers with branching inside the epidermis were considered one nerve. IENFDs

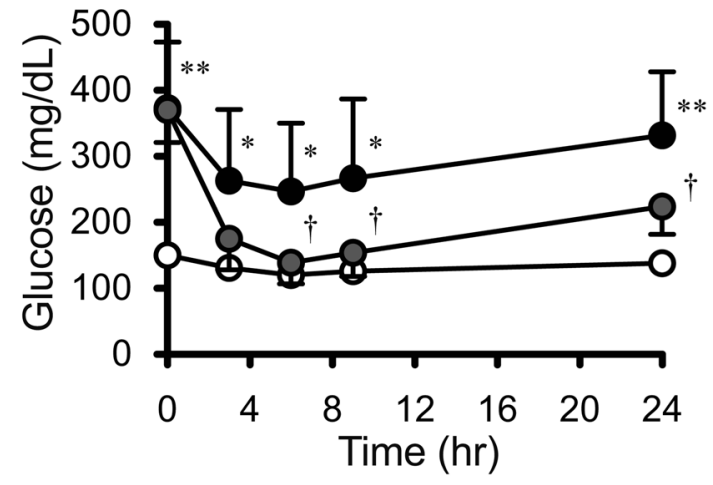

Fig. 1. Hypoglycemic effect of phlorizin in SDT fatty rats. A single dose of phlorizin clearly decreased blood glucose levels. Six hours after administration, blood glucose reached normal levels. At 24 hours, the effect of phlorizin partially remained. White circles; SD rats, black circles; SDT fatty rats (vehicle), gray circles; SDT fatty rats (100 $\mathrm{mg} / \mathrm{kg}$ phlorizin). Each value represents the mean $\pm \mathrm{SD}$ $(\mathrm{n}=4) .{ }^{* *} P<0.01,{ }^{*} P<0.05$ vs. age-matched SD rats. ${ }^{\dagger} P<0.05$ vs. vehicle treated SDT fatty rats (unpaired $t$-test).

were expressed as numbers of epidermal nerve fibers per length of the epidermal basement membrane (fibers $/ \mathrm{mm}$ ).

\section{Statistical analysis}

Results are expressed as the mean \pm SD. Statistical analyses of differences between mean values were performed using an F-test, followed by a Student's $t$-test or Aspin-Welch's $t$-test. A Wilcoxon rank-sum test was used for cataract scores. All statistical analyses were performed using the EXSUS statistical analysis system for biological experiment data (CAC EXICARE, Tokyo, Japan). Differences were accepted as significant at $P<0.05$.

\section{Results}

\section{Hypoglycemic effect of phlorizin on SDT fatty rats}

To confirm the dose of phlorizin for the study, $100 \mathrm{mg} /$ $\mathrm{kg}$ of phlorizin was subcutaneously administered once to 18 week-old female SDT fatty rats under non-fasted conditions (Fig. 1). Prior to phlorizin treatment, the blood glucose level in SDT fatty rats was $370 \pm 49 \mathrm{mg} / \mathrm{dl}$. Six hours after dosing, the blood glucose level in the phlorizin treated group decreased to an almost normal level (139 \pm $32 \mathrm{mg} / \mathrm{dl})$. The hypoglycemic effect of phlorizin was sustained for $24 \mathrm{~h}(224 \pm 42 \mathrm{mg} / \mathrm{dl})$; although the effect of phlorizin was weakened at $24 \mathrm{~h}$, normal blood glucose levels were expected with repeat dosing. Therefore, a 
A
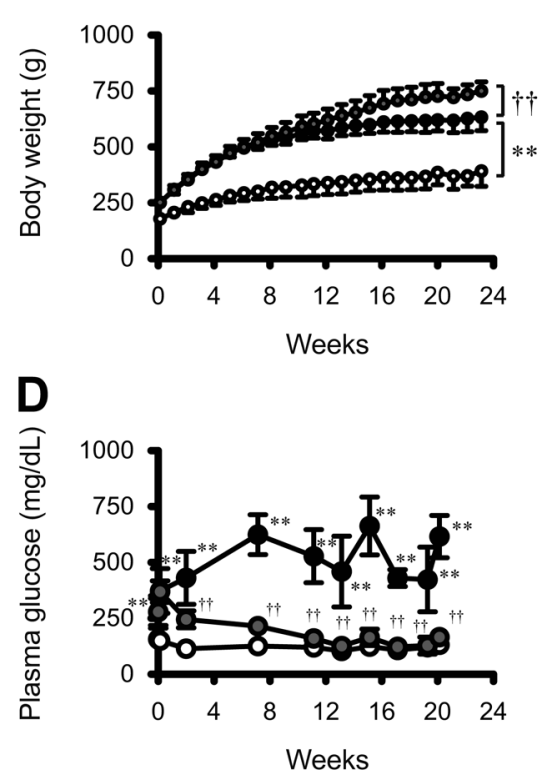

G

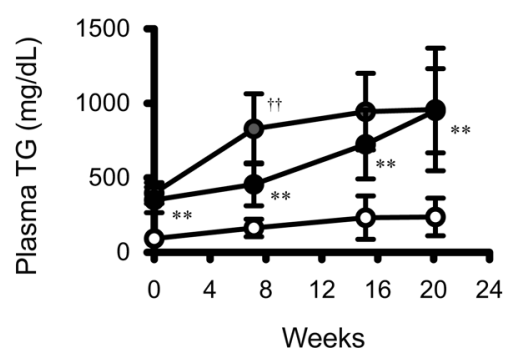

B

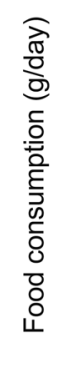

E

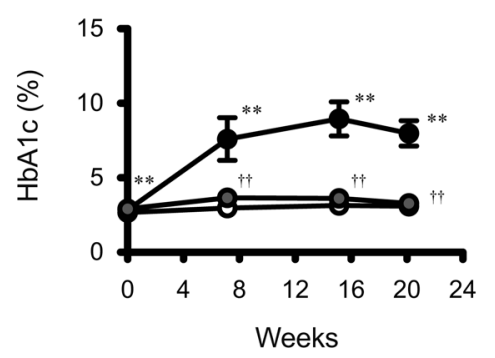

H

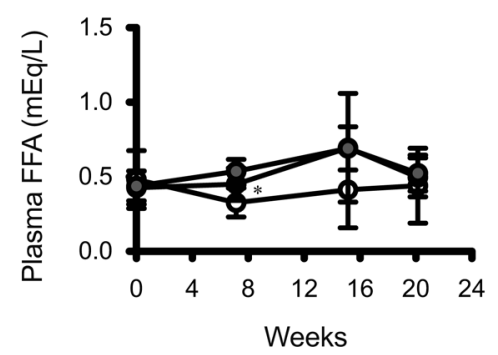

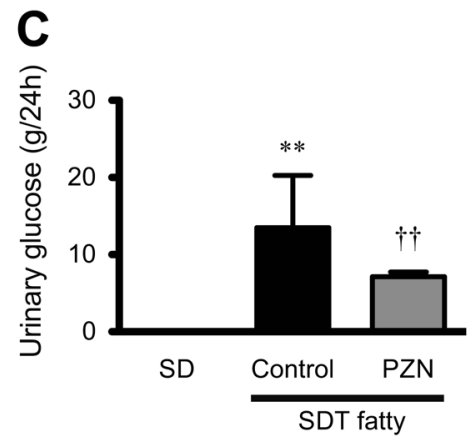

$\mathbf{F}$

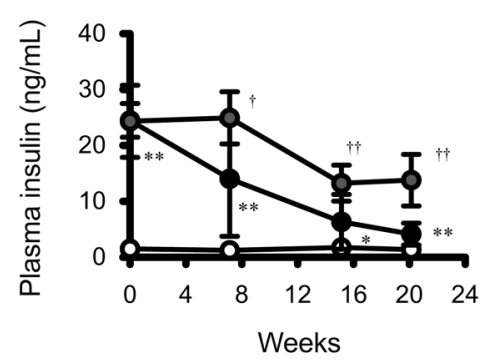

I

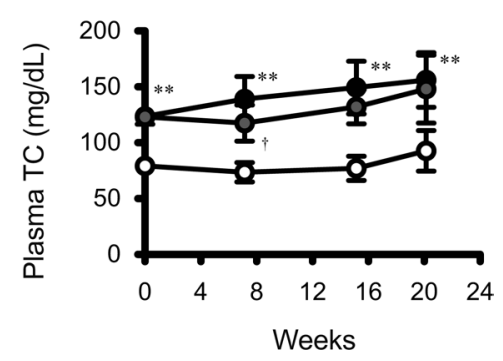

Fig. 2. Effect of phlorizin on biochemical parameters of SDT fatty rats. Effects of phlorizin on (A) body weight, (B) food consumption (at the end of experiment; 29 weeks of age), (C) Urinary glucose, (D) plasma glucose levels, (E) blood HbA1c levels, (F) plasma insulin levels, (G) plasma triglyceride levels, (H) plasma free fatty acid levels and (I) plasma total cholesterol levels. Phlorizin treatment improved hyperglycemia and delayed insulin level deceases. Hyperlipidemia was not clearly prevented with phlorizin. White circles; SD rats, black circles; SDT fatty rats (vehicle), gray circles; SDT fatty rats (100 mg/ $\mathrm{kg}$ phlorizin). Each value represents the mean $\pm \mathrm{SD}(\mathrm{n}=8) .{ }^{* *} P<0.01,{ }^{*} P<0.05$ vs. age-matched SD rats. ${ }^{\dagger} P<0.01,{ }^{\dagger} P<0.05$ vs. vehicle treated SDT fatty rats (unpaired $t$-test).

single dose of $100 \mathrm{mg} / \mathrm{kg}$ phlorizin is sufficient to reduce plasma glucose level for $24 \mathrm{~h}$ in female SDT fatty rats.

\section{Effect of phlorizin on biochemical parameters of SDT fatty rats}

Body weights of SDT fatty rats were significantly higher than SD rats in the experiment. Phlorizin-treated SDT fatty rats were heavier than vehicle-treated SDT fatty rats after 12 weeks (Fig. 2A). At the end of the study, food consumption of SDT fatty rats was almost twice that of SD rats; however, there were no differences between the vehicle treated group and phlorizin treated group (Fig. 2B). Urinary glucose considerably increased in SDT fatty rats, and phlorizin treatment significantly decreased glucose excretion (Fig. 2C).

After 2 weeks, blood glucose levels of SDT fatty rats increased to a range of $424 \mathrm{mg} / \mathrm{dl}$ to $663 \mathrm{mg} / \mathrm{dl}$ (Fig. 2D). Blood glucose levels were adequately controlled with phlorizin treatment during the experiment and gradually decreased to near normal range $(123 \mathrm{mg} / \mathrm{dl}$ to $167 \mathrm{mg} / \mathrm{dl})$. Blood glucose levels of normal SD rats were in the range of $104 \mathrm{mg} / \mathrm{dl}$ to $157 \mathrm{mg} / \mathrm{dl}$. HbAlc levels reflected the change of blood glucose level accurately (Fig. 2E). Plasma insulin levels of SDT fatty rats were signifi- 
cantly higher than SD rats at the beginning of the experiment ( 6 weeks of age) and gradually decreased to normal level at 20 weeks. Phlorizin treatment delayed insulin decreases and insulin was kept high even at the end of study (Fig. 2F). Plasma TG levels (Fig. 2G), FFA levels (Fig. 2H) and TC levels (Fig. 2I) in SDT fatty rats were higher than SD rats. TG levels and FFA levels in the phlorizin treated group were significantly higher and TC levels were lower than the vehicle treated group only at 7 weeks treatment.

Effect of phlorizin on diabetic nephropathy (DN) of SDT fatty rats

After 20 weeks of treatment with phlorizin, urinary parameters were evaluated to assess the effects of phlorizin on DN. Urinary albumin excretion (UAE; Fig. 3A) considerably increased in SDT fatty rats. Creatinine clearance (Fig. 3B) and urinary 8-OHdG (Fig. 3C) also increased significantly. Creatinine clearance decreased significantly with phlorizin treatment; however, the effects on UAE and 8-OHdG were limited.

Figures 3D and 3E show the typical microphotographs of the kidneys of SD rats and SDT fatty rats. Slight glomerulosclerosis and tubular regeneration, dilation, Armanni-Ebstein changes and inflammatory cell infiltration in interstitial areas were found in SDT fatty rats. Phlorizin treatment prevented Armanni-Ebstein changes (Fig. $3 \mathrm{~F}$ ); however, other renal abnormal findings did not recover with controlled blood glucose level. Moreover, tubular dilation and inflammatory cell infiltration in the urothelium were frequently observed in phlorizin treated SDT fatty rats.

Effect of phlorizin on diabetic peripheral neuropathy (DPN) of SDT fatty rats

At the end of the treatment, sciatic MNCV and SNCV were measured under anesthesia. Both MNCV and SNCV were significantly delayed in SDT fatty rats compared with non-diabetic SD rats. The MNCV of SDT fatty rats decreased to $82.0 \%$ of that in SD rats and SNCV decreased to $82.4 \%$ (Figs. $4 \mathrm{~A}$ and B). These functional impairments in nerves were corrected to $95.6 \%$ and $98.3 \%$ of those in normal rats, respectively, with 23-weeks of phlorizin administration.

To evaluate peripheral nerve density, skin biopsies with quantification of IENFD were performed. IENFD from the skin of the hind paw foot of SDT fatty rats decreased significantly at 29 weeks of age (SD rats; 27.1 \pm 2.3 fibers $/ \mathrm{mm}$, SDT fatty rats; $14.8 \pm 3.1$ fibers $/ \mathrm{mm}$ ). 23 weeks of phlorizin treatment prevented the decrease of nerve fibers $(23.6 \pm 3.2$ fibers $/ \mathrm{mm})$ (Figs. $4 \mathrm{C}-\mathrm{F}$ ).

\section{Effect of phlorizin on diabetic retinopathy $(D R)$ and cataracts of SDT fatty rats}

At 29 weeks of age, SDT fatty rats showed prolongations of peak latencies of oscillatory potential in ERGs compared with age-matched SD rats. There was a significant prolongation of peak latency for each individual oscillatory potential $\left(\mathrm{OP}_{1}-\mathrm{OP}_{3}\right)$, as well as that of the summed potential $\Sigma\left(\mathrm{OP}_{1}-\mathrm{OP}_{3}\right)$. Prolongation of these peak latencies decreased significantly with 23 weeks of phlorizin administration (Fig. 5A). Hisopathologically, retinal folding was observed in SDT fatty rats as previously reported [17, 23]. Retinal abnormalities were completely prevented with phlorizin (Figs. 5C-E).

Cataracts progressed in SDT fatty rats from 8 weeks of age. At 29 weeks of age, all SDT fatty rats showed mature cataracts via macroscopic observation. Cataracts in phlorizin treated SDT fatty rats did not progress even at the end of experiments (Figs. 5B and $\mathrm{F}-\mathrm{H}$ ).

\section{Discussion}

The SDT fatty rat has been developed as a new type 2 diabetes model with rapidly progressing diabetic microvascular complications $[17,19,20]$. SDT fatty rats develop diabetes from 5 weeks of age, and the incidence of diabetes in both male and female SDT fatty rats is $100 \%$. Previously, we evaluated some hypoglycemic drugs such as pioglitazone, metformin and DPP IV inhibitor, on male SDT fatty rats $[4,29]$. Despite that the female SDT fatty rats also show severe diabetes and its complications, only few experiments have been reported [29]. Therefore, in the present study, we used female SDT fatty rats to investigate the characteristics of diabetic complications in this animal model by controlling blood glucose level with phlorizin. We have confirmed that phlorizin treatment sufficiently reduce blood glucose level in male SDT rats (unpublished data). Phlorizin is a non-selective SGLT inhibitor and recently-launched SGLT2 inhibitors are treated as anti-hyperglycemic drugs with a novel mechanism of action. Although it is difficult to distinguish the effect on SGLT1 in gut from that on SGLT2 in kidney, the changes in body weight, blood glucose levels and HbA1c suggest the usefulness of SGLT inhibitor phlorizin in the treatment of hyperglycemia. By using phlorizin, 
A
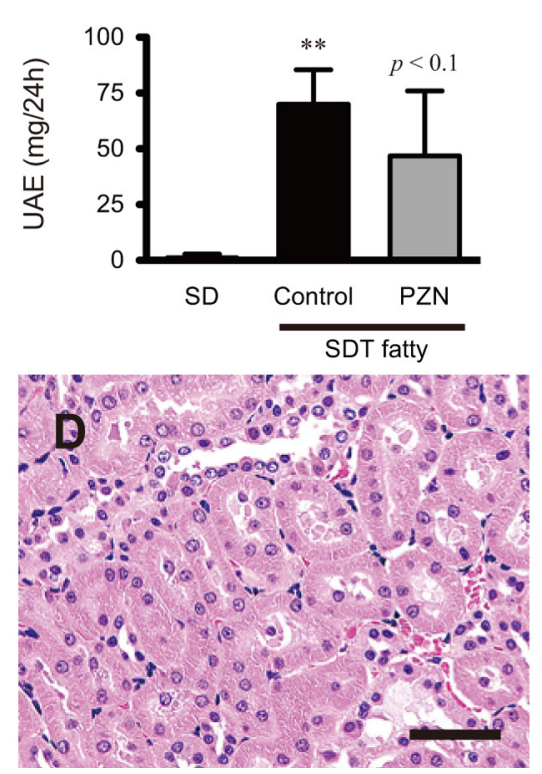

B
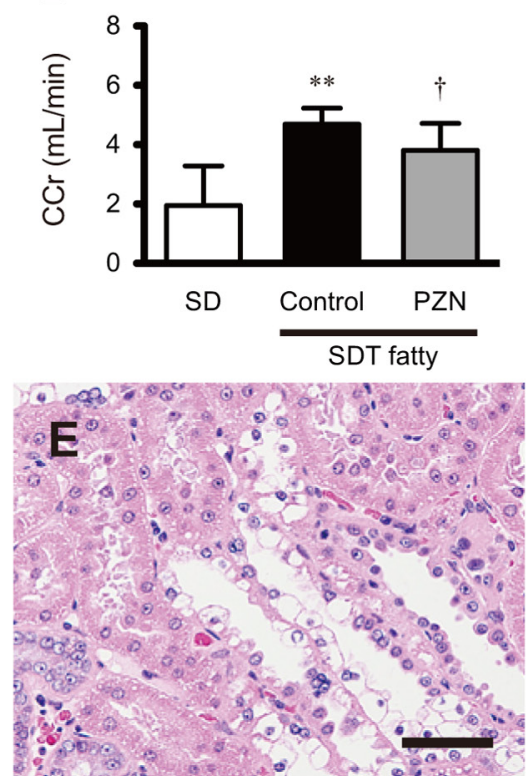

C
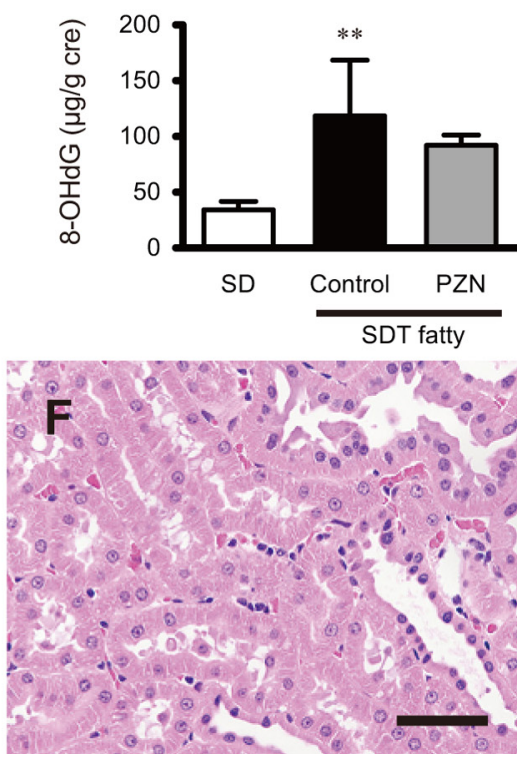

Fig. 3. Effect of phlorizin on diabetic nephropathy of SDT fatty rats. Urinary albumin excretion (A), creatinine clearance (B) and urinary 8-OHdG $(\mathrm{C})$ increased in SDT fatty rats. Increases in all these renal parameters were partially prevented with phlorizin treatment. Typical microphotographs of the kidneys of SD rats (D) and SDT fatty rats (E). Tubular dilation and Armanni-Ebstein changes were found in SDT fatty rats. Phlorizin treatment completely prevented these tubular abnormalities (F). Bars $=50 \mu \mathrm{m}$. Each value represents the mean $\pm \mathrm{SD}(\mathrm{n}=8) .{ }^{* *} P<0.01$ vs. age-matched SD rats, ${ }^{\dagger} P<0.05$ vs. vehicle treated SDT fatty rats (unpaired $t$-test).

A
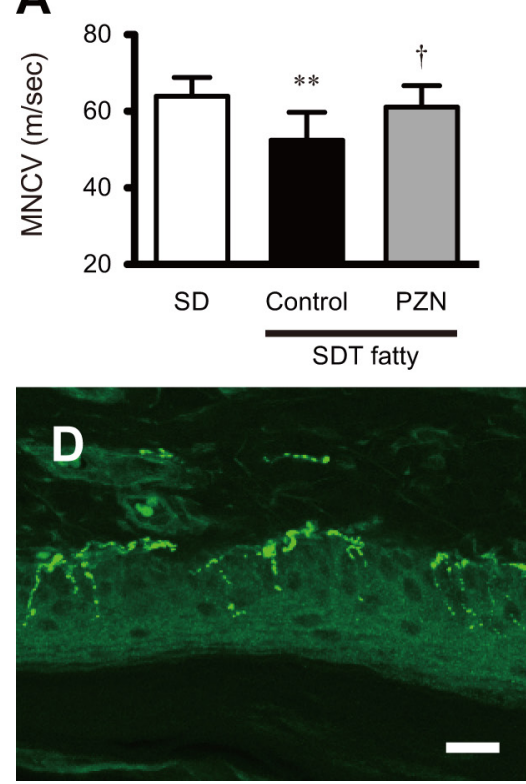

B
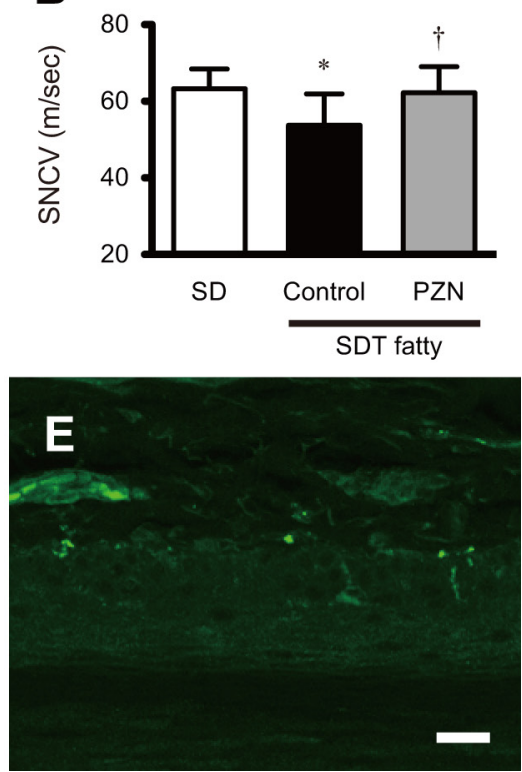

C
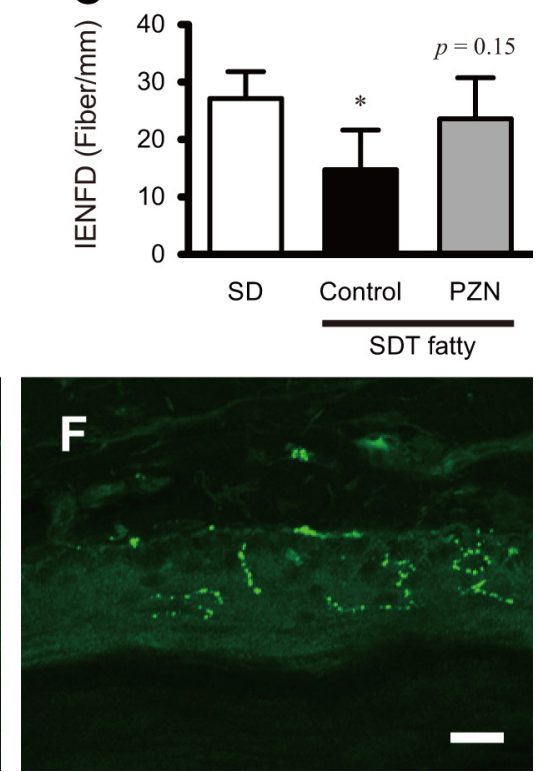

Fig. 4. Effect of phlorizin on diabetic peripheral neuropathy of SDT fatty rats. The effects of phlorizin on peripheral nerve dysfunction (sciatic MNCV and SNCV) and IENFD in female SDT fatty rats were evaluated. Impairments of MNCV (A) and SNCV (B) improved significantly with phlorizin treatment compared with vehicle-treated SDT fatty rats. Reductions in IENFD were also prevented with phlorizin (C). Typical confocal microscopic pictures of anti-PGP9.5 immunostained hind paw skin from SD rats (D), vehicle-treated SDT fatty rats (E), and phlorizin-treated SDT fatty rats $(\mathrm{F})$. Bars $=20 \mu \mathrm{m}$. Each value represents the mean $\pm \mathrm{SD}(\mathrm{n}=6-8) .{ }^{* *} P<0.01,{ }^{*} P<0.05$ vs. age-matched SD rats, ${ }^{\dagger} P<0.05$ vs. vehicle treated SDT fatty rats (unpaired $t$-test). 
A

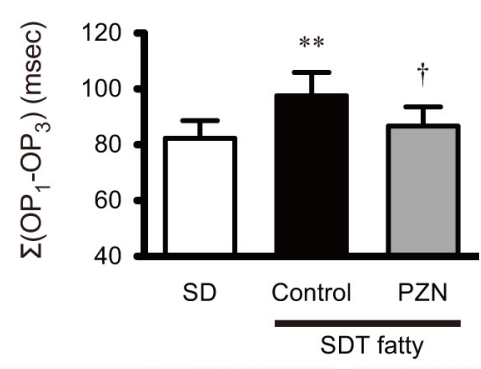

B

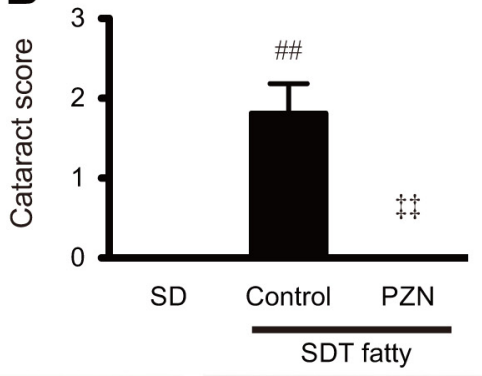

E

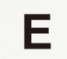

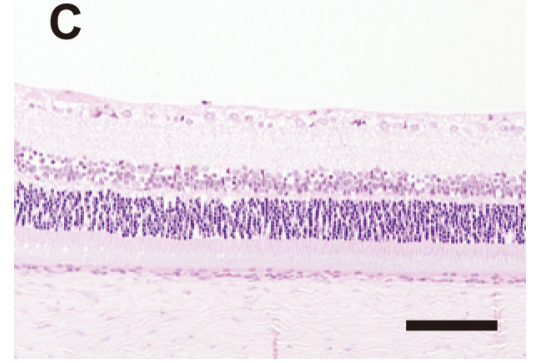
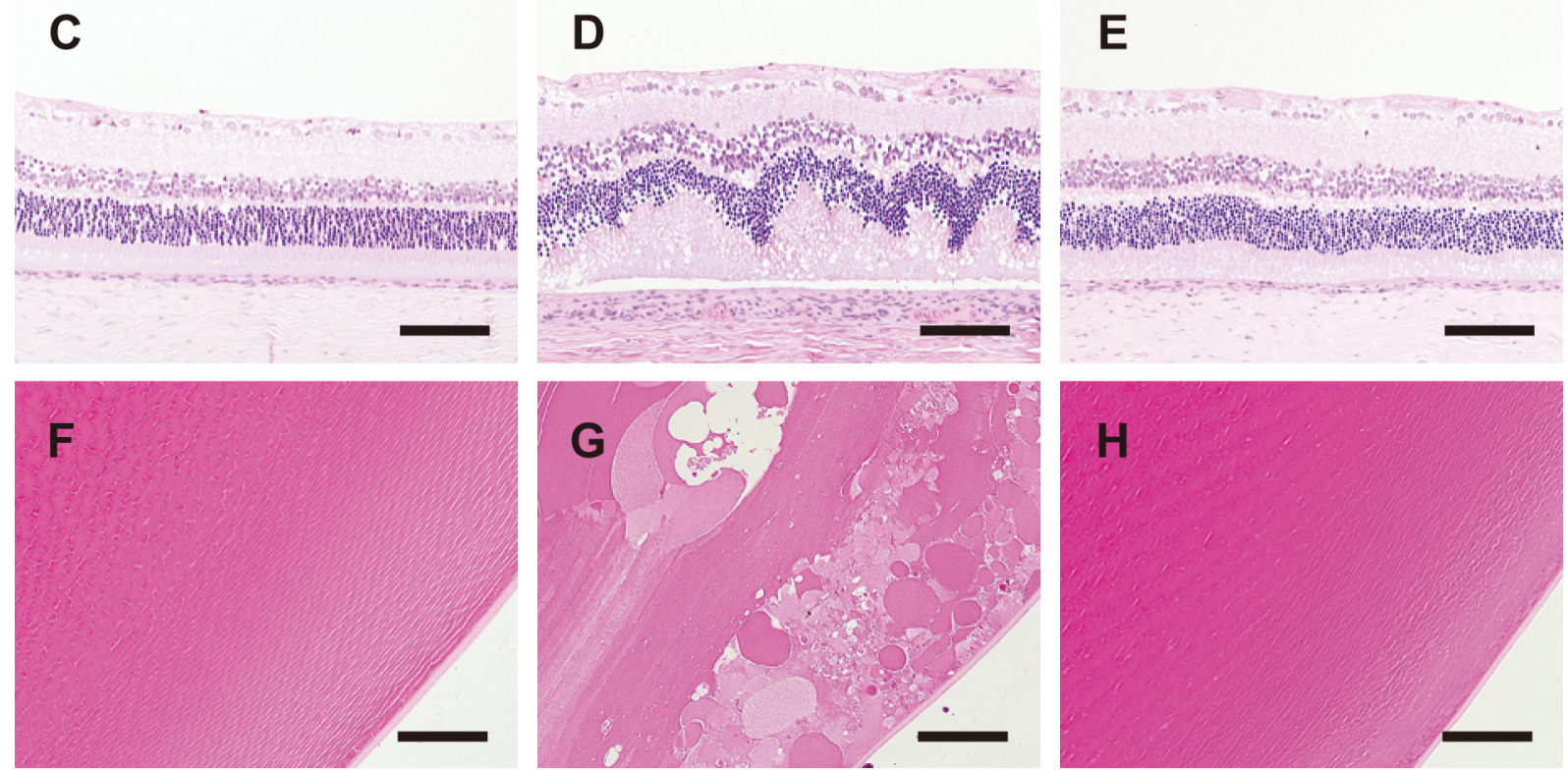

Fig. 5. Effect of phlorizin on diabetic retinopathy and cataracts of SDT fatty rats. Delayed OPs in ERG were observed in SDT fatty rats (A). Cataracts also progressed in SDT fatty rats (B). These eye disorders were prevented with phlorizin treatment. Compared to SD rats (C), retinal folding and thickening were observed in SDT fatty rats (D) and were improved with phlorizin (E). Bars $=100 \mu \mathrm{m}$. Normal rats showed clear lens (F), but mature cataracts were found in SDT fatty rats (G). Cataracts did not progress in phlorizin treated SDT fatty rats $(\mathrm{H})$. Bars $=50 \mu \mathrm{m}$. Each value represents the mean $\pm \operatorname{SD}(\mathrm{n}=8) .{ }^{* *} P<0.01$ vs. age-matched SD rats, ${ }^{\dagger} P<0.05$ vs. vehicle treated SDT fatty rats (unpaired $t$-test). ${ }^{\#} P<0.01$ vs. age-matched SD rats, $\$ P<0.01$ vs. vehicle treated SDT fatty rats (Wilcoxon rank-sum test).

primarily hypoglycemic effects are expected without affecting other biochemical parameters. Pharmacological effects of phlorizin on diabetic animal models were reported previously. In concurrence with our result, phlorizin treatment prevented hyperglycemia and preserved insulin mRNA levels but failed to prevent hypertriglyceridemia in ZDF rats [8].

Body weights of SDT fatty rats are obviously heavier than normal SD rats because of hyperphagia due to the lack of leptin signaling $[19,20]$. With the progress of hyperglycemia, body weight gain gradually slowed down. Repeated treatment with phlorizin completely prevented the increase of plasma glucose levels during the experiment. Improvement of hyperglycemia with phlorizin is considered to influence general condition, leading to further increases in body weight.

For the kidneys of SDT fatty rats, we previously reported histopathological changes in glomeruli (glomerulosclerosis, increased mesangial matrix and glomerular hypertrophy, and nodular lesions) and renal tubules (glycogen deposition (Armanni-Ebstein lesions), fibrosis, inflammatory cell filtration and tubular dilation) $[14,17,20]$. In the present study, SDT fatty rats showed significant increases in urinary glucose, UAE, and creatinine clearance. The primary effect of phlorizin is inducing glucosuria; however, urinary glucose levels in the phlorizin treated group were lower than the vehicle treated group. Lower glucosuria may reflect reduced 
plasma glucose levels in phlorizin treated SDT fatty rats at these time points. These data are consistent with previous report that hyperglycemia was decreased by phlorizin in streptozotocin (STZ)-induced diabetes rats but significant diuresis and glucosuria remain [24]. Although creatinine clearance decreased significantly, UAE and 8-OHdG were partially decreased with phlorizin treatment; these parameters did not recover completely by controlling only blood glucose level. In addition to hyperglycemia, abnormal lipid metabolism has been considered an important factor in the pathogenesis of DN $[7,10,28]$. Since phlorizin treatment failed to reduce blood TG and TC levels, uncontrolled dyslipidemia may affect DN in SDT fatty rats. In the report using STZ rats, phlorizin prevented proteinuria, hyperfiltration and whole kidney hypertrophy, but not glomerular hypertrophy. Therefore, some part of renal impairment in STZ rats is uncontrollable with decreasing blood glucose level alone [18]. On the other hand, some histopathological findings were observed in the phlorizin-treated group. Increased urine volume and susceptibility to urinary tract infection by inhibiting SGLT2 in proximal renal tubules may lead to tubular dilation and inflammatory cell infiltration in the urothelium of phlorizin treated SDT fatty rats.

We reported that caudal MNCV in both male and female SDT fatty rats was delayed at 24 weeks of age $[16,29]$. Histopathologically, at 40 weeks of age, significant decreases in sural nerve fiber number due to atrophy were observed in male SDT fatty rats [29]. In the present study, we evaluated IENFD in SDT fatty rats as a hallmark of small fiber neuropathy. IENFD is considered a marker of DPN that reduces from early stage diabetes [27]. Similar to other diabetic animal models $[2,9]$, SDT fatty rats showed significantly decreased IENFD. Significant histopathological and functional preservation of peripheral nerves were achieved with phlorizin. As is the case with DN, effects of dyslipidemia and hypertension on DPN have been pointed out $[5,22]$. In contrast to $\mathrm{DN}$, these factors have insignificant effects on DPN in SDT fatty rats because controlling only blood glucose level prevented histopathological and functional nerve impairments.

As a marker of DR, retinal function was evaluated using ERGs. Previously, we reported the ERG results in SDT fatty rats; prolongation of OPs was observed in both males (16 weeks of age) and females (22 weeks of age) $[16,20]$. Delayed OPs were also observed in the present study and phlorizin treatment prevented retinal dysfunction. Histopathological changes in the lens, such as hyperplasia of the epithelium, vacuolation of fibers, and formation of Morgagnian globules, coincide with previous reports $[14,20]$. Furthermore, retinal lesions, such as folding and thickening, which are found in aged SDT fatty rats $[16,17]$, were also observed. These histopathological abnormalities were not observed in the eyes of SDT fatty rats treated with phlorizin. Although dyslipidemia is a key factor of DR [6], our findings suggest that hyperglycemia is the major cause of DR and other ocular changes in SDT fatty rats.

In conclusion, diabetic complications in SDT fatty rats are caused by sustained severe hyperglycemia. Notably, other factors than hyperglycemia, such as hyperlipidemia and hypertension may be involved in diabetic nephropathy in SDT fatty rats. In addition, we reported that enhanced vascular endothelial growth factor (VEGF) signaling also contributes to microvascular dysfunction in SDT rats recently [21]. Clarifying the mechanism of diabetic complications further enhances the prospects for the usefulness of this animal model in developing new drugs and therapies for diabetic microvascular complications.

\section{References}

1. Abdul-Ghani, M.A. and DeFronzo, R.A. 2008. Inhibition of renal glucose reabsorption: a novel strategy for achieving glucose control in type 2 diabetes mellitus. Endocr. Pract. 14: 782-790. [Medline] [CrossRef]

2. Brussee, V., Guo, G., Dong, Y., Cheng, C., Martinez, J.A., Smith, D., Glazner, G.W., Fernyhough, P., and Zochodne, D.W. 2008. Distal degenerative sensory neuropathy in a long-term type 2 diabetes rat model. Diabetes 57: 16641673. [Medline] [CrossRef]

3. Ehrenkranz, J.R., Lewis, N.G., Kahn, C.R., and Roth, J. 2005. Phlorizin: a review. Diabetes Metab. Res. Rev. 21: 31-38. [Medline] [CrossRef]

4. Fukuda, S., Miyajima, K., Sasase, T., and Ohta, T. 2011. Spontaneously Diabetic Torii Lepr ${ }^{f a}$ (SDT Fatty) rat: A novel model of obese type 2 diabetes. Open Diabetes J. 4: 30-36. [CrossRef]

5. Goncalves, A.C., Tank, J., Diedrich, A., Hilzendeger, A., Plehm, R., Bader, M., Luft, F.C., Jordan, J., and Gross, V. 2009. Diabetic hypertensive leptin receptor-deficient $\mathrm{db} / \mathrm{db}$ mice develop cardioregulatory autonomic dysfunction. $\mathrm{Hy}$ pertension 53: 387-392. [Medline] [CrossRef]

6. Chew, E.Y., Ambrosius, W.T., Davis, M.D., Danis, R.P., Gangaputra, S., Greven, C.M., Hubbard, L., Esser, B.A., Lovato, J.F., Perdue, L.H., Goff, D.C. Jr., Cushman, W.C., Ginsberg, H.N., Elam, M.B., Genuth, S., Gerstein, H.C., 
Schubart, U., Fine, L.J., ACCORD Study Group ACCORD Eye Study Group 2010. Effects of medical therapies on retinopathy progression in type 2 diabetes. N. Engl. J. Med. 363: 233-244. [Medline] [CrossRef]

7. Guijarro, C., Kasiske, B.L., Kim, Y., O’Donnell, M.P., Lee, H.S., and Keane, W.F. 1995. Early glomerular changes in rats with dietary-induced hypercholesterolemia. Am. J. Kidney Dis. 26: 152-161. [Medline] [CrossRef]

8. Harmon, J.S., Gleason, C.E., Tanaka, Y., Poitout, V., and Robertson, R.P. 2001. Antecedent hyperglycemia, not hyperlipidemia, is associated with increased islet triacylglycerol content and decreased insulin gene mRNA level in Zucker diabetic fatty rats. Diabetes 50: 2481-2486. [Medline] [CrossRef]

9. Himeno, T., Kamiya, H., Naruse, K., Harada, N., Ozaki, N., Seino, Y., Shibata, T., Kondo, M., Kato, J., Okawa, T., Fukami, A., Hamada, Y., Inagaki, N., Seino, Y., Drucker, D.J., Oiso, Y., and Nakamura, J. 2011. Beneficial effects of exendin-4 on experimental polyneuropathy in diabetic mice. Diabetes 60: 2397-2406. [Medline] [CrossRef]

10. Hsu, C.Y., Bates, D.W., Kuperman, G.J., and Curhan, G.C. 2000. Diabetes, hemoglobin $\mathrm{A}(1 \mathrm{c})$, cholesterol, and the risk of moderate chronic renal insufficiency in an ambulatory population. Am. J. Kidney Dis. 36: 272-281. [Medline] [CrossRef]

11. International Diabetes Federation 2011. IDF Diabetes Atlas, 5th ed., Brussels, Belgium.

12. Ishii, Y., Maki, M., Yamamoto, H., Sasase, T., Kakutani, M., and Ohta, T. 2010. Evaluation of blood pressure in spontaneously diabetic torii-Lepr ${ }^{f a}$ rats. Exp. Anim. 59: 525-529. [Medline] [CrossRef]

13. Ishii, Y., Maki, M., Yamamoto, H., Sasase, T., Kakutani, M., and Ohta, T. 2011. Blood pressure characteristics of female spontaneously diabetic Torii-Lepr ${ }^{f a}$ rats. J. Vet. Med. Sci. 73: 501-505. [Medline] [CrossRef]

14. Ishii, Y., Ohta, T., Sasase, T., Morinaga, H., Ueda, N., Hata, T., Kakutani, M., Miyajima, K., Katsuda, Y., Masuyama, T., Shinohara, M., and Matsushita, M. 2010. Pathophysiological analysis of female spontaneously diabetic Torii fatty rats. Exp. Anim. 59: 73-84. [Medline] [CrossRef]

15. Joint Task Force of the EFNS and the PNS 2010. European Federation of Neurological Societies/Peripheral Nerve Society Guideline on the use of skin biopsy in the diagnosis of small fiber neuropathy. Report of a joint task force of the European Federation of Neurological Societies and the Peripheral Nerve Society. J. Peripher. Nerv. Syst. 15: 79-92. [Medline] [CrossRef]

16. Katsuda, Y., Ohta, T., Miyajima, K., Kemmochi, Y., Sasase, T., Tong, B., Shinohara, M., and Yamada, T. 2014. Diabetic complications in obese type 2 diabetic rat models. Exp. Anim. 63: 121-132. [Medline] [CrossRef]

17. Kemmochi, Y., Fukui, K., Maki, M., Kimura, S., Ishii, Y., Sasase, T., Miyajima, K., and Ohta, T. 2013. Metabolic disorders and diabetic complications in spontaneously diabetic Torii Lepr ${ }^{\text {fa }}$ rat: a new obese type 2 diabetic model. J Diabetes Res. 2013: 948257. [Medline]

18. Malatiali, S., Francis, I., and Barac-Nieto, M. 2008. Phlorizin prevents glomerular hyperfiltration but not hypertrophy in diabetic rats. Exp. Diabetes Res. 2008: 305403. [Medline]
[CrossRef]

19. Masuyama, T., Katsuda, Y., and Shinohara, M. 2005. A novel model of obesity-related diabetes: introgression of the Lepr ${ }^{f a}$ allele of the Zucker fatty rat into nonobese spontaneously diabetic Torii (SDT) rats. Exp. Anim. 54: 13-20. [Medline] [CrossRef]

20. Matsui, K., Ohta, T., Oda, T., Sasase, T., Ueda, N., Miyajima, K., Masuyama, T., Shinohara, M., and Matsushita, M. 2008. Diabetes-associated complications in spontaneously diabetic Torii fatty rats. Exp. Anim. 57: 111-121. [Medline] [CrossRef]

21. Mukai, E., Ohta, T., Kawamura, H., Lee, E.Y., Morita, A., Sasase, T., Miyajima, K., Inagaki, N., Iwanaga, T., and Miki, T. 2014. Enhanced vascular endothelial growth factor signaling in islets contributes to $\beta$ cell injury and consequential diabetes in spontaneously diabetic Torii rats. Diabetes Res. Clin. Pract. 106: 303-311. [Medline] [CrossRef]

22. Obrosova, I.G., Ilnytska, O., Lyzogubov, V.V., Pavlov, I.A., Mashtalir, N., Nadler, J.L., and Drel, V.R. 2007. High-fat diet induced neuropathy of pre-diabetes and obesity: effects of "healthy" diet and aldose reductase inhibition. Diabetes 56 : 2598-2608. [Medline] [CrossRef]

23. Ohta, T., Katsuda, Y., Miyajima, K., Sasase, T., Kimura, S., Tong, B., and Yamada, T. 2014. Gender differences in metabolic disorders and related diseases in spontaneously diabetic Torii-Lepr ${ }^{f a}$ rats. J Diabetes Res 2014: 841957. [Medline] [CrossRef]

24. Osorio, H., Coronel, I., Arellano, A., Pacheco, U., Bautista, R., Franco, M., and Escalante, B. 2012. Sodium-glucose cotransporter inhibition prevents oxidative stress in the kidney of diabetic rats. Oxid. Med. Cell. Longev. 2012: 542042. [Medline] [CrossRef]

25. Sasase, T., Morinaga, H., Abe, T., Miyajima, K., Ohta, T., Shinohara, M., Matsushita, M., and Kakehashi, A. 2009. Protein kinase $\mathrm{C}$ beta inhibitor prevents diabetic peripheral neuropathy, but not histopathological abnormalities of retina in spontaneously diabetic Torii rat. Diabetes Obes. Metab. 11: 1084-1087. [Medline] [CrossRef]

26. Sasase, T., Ohta, T., Ogawa, N., Miyajima, K., Ito, M., Yamamoto, H., Morinaga, H., and Matsushita, M. 2006. Preventive effects of glycaemic control on ocular complications of spontaneously diabetic Torii rat. Diabetes Obes. Metab. 8: 501-507. [Medline] [CrossRef]

27. Shun, C.T., Chang, Y.C., Wu, H.P., Hsieh, S.C., Lin, W.M., Lin, Y.H., Tai, T.Y., and Hsieh, S.T. 2004. Skin denervation in type 2 diabetes: correlations with diabetic duration and functional impairments. Brain 127: 1593-1605. [Medline] [CrossRef]

28. Sun, L., Halaihel, N., Zhang, W., Rogers, T., and Levi, M. 2002. Role of sterol regulatory element-binding protein 1 in regulation of renal lipid metabolism and glomerulosclerosis in diabetes mellitus. J. Biol. Chem. 277: 18919-18927. [Medline] [CrossRef]

29. Yamaguchi, T., Sasase, T., Mera, Y., Tomimoto, D., Tadaki, H., Kemmochi, Y., Ohta, T., Sato, E., and Matsushita, M. 2012. Diabetic peripheral neuropathy in spontaneously diabetic Torii-Lepr ${ }^{f a}$ (SDT fatty) rats. J. Vet. Med. Sci. 74: 1669-1673. [Medline] [CrossRef] 